\title{
Rationally designed peptidomimetics
}

Andrew Jamieson

University of Glasgow, United Kingdom

https://doi.org/10.17952/35EPS.2018.035

Peptides are well known to act as potent and selective ligands for a variety of bimolecular targets. Although there are a many peptide drugs in clinical use, their potential has been limited by unfavorable physicochemical properties including limited plasma stability and a lack of cell permeability. Peptidomimetics are molecules that can be rationally designed to overcome many of the issues associated with peptides and provide tool molecules for chemical biology and lead compounds for drug discovery.[1]

Disulfide bonds play an important structural and/or catalytic role in many proteins. However the synthesis of peptides containing more then one disulfide-bond is not trivial.[2] Solid phase synthesis of the linear peptide and subsequent oxidative folding under thermodynamic conditions generally produces a mixture of structural isomers. Synthesis of specific isomers requires a solid phase approach and orthogonal protection strategy. Disulfide bond rich peptides and proteins are susceptible to disulfide bond shuffling and reduction catalysed by glutathione. As such, an effective surrogate that accurately mimics a disulfide bond would be a powerful tool in chemical biology with a range of applications from understanding protein-folding mechanisms to peptide drug discovery.

A number of synthetic disulfide bond mimetics have been developed. The most successful strategies to date include diselenide (Se-Se), selenylsulfide ( $\mathrm{Se}-\mathrm{S})$, cystathionine $(\mathrm{CH} 2-\mathrm{S}$, Ctt), and ditelluride (Te-Te) bonds. However, each of these methods has disadvantages such as multiple step synthesis required for their preparation such that they have not be adopted up by the peptide community. Other methods to mimic disulfide bonds include olefin-based isosteres and thioethers. However, thioethers require multistep synthesis utilizing complex orthogonal protecting group strategies. Olefin-based isosteres produced by ring-closing metathesis (RCM) reactions give complex mixtures of cis/trans isomers, and required a subsequent palladium-catalyzed hydrogenation step to access a suboptimal, conformationally flexible alkane bridge. Kolmer was first to describe the use of a 1,5-disubstitiuted 1,2,3-triazole as a disulfide bond mimic.[3] In this seminal work the disulfide bond within a macrocyclic sunflower trypsin inhibitor-I was replaced by a 1,5 triazole and the resulting peptidomimetic retained biological activity.

Urotensin-II (U-II) is the endogenous cyclic peptide ligand of the urotensin receptor (UTR).[4] The U-II peptide is the most potent vasoconstrictor known and so is a promising lead compound that targets the UTR for development as a potential therapeutic for the treatment of heart failure and atherosclerosis. U-II is an eleven-residue peptide that adopts a bioactive beta-turn conformation that is constrained by a crossstrand disulfide bond. An issue with studying the interaction of U-II with the UTR is that the peptide acts as a pseudo-irreversible agonist. Previously developed peptide ligands are based on U-II and so display the undesired effect of irreversibility. The development of reversible UTR ligands would be a useful addition to the biochemical toolbox to investigate the physiological role of the U-II/UTR system. 


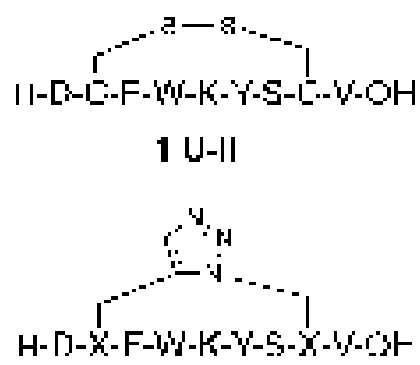

2 I,

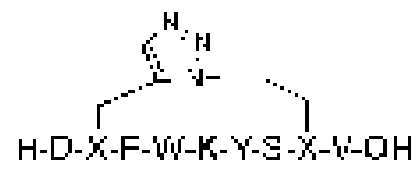

31.5 triil<c:<smiles>[AlH2]n1cc(C[Al][TeH])nn1</smiles>

4 1,4-tiliajzoli)

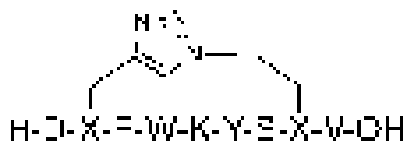

5 1.4-liazc в

Figure 1: Urotensin-II peptide 1 and peptidomimetic analogues 2-5.

A series of U-II analogues 2-5 were designed to incorporate either a 1,4- or 1,5-triazole bridge as a disulfide bond surrogate (Figure 1).[5] The four analogues 2-5 were assessed by computationaloverlay with the U-II disulfide bond. Compound 3 incorporating a 1,5-triazole and 6-atom bridge proved to be the most accurate surrogate for the disulfide bridge, giving a room-mean-square-deriation (RMSD) of $0.182 \AA$. The linear peptides required to produce the peptidomimetics were synthesised by automated microwave assisted solid phase peptide synthesis using a Fmoc/tBu protection strategy. Commercially available building blocks Fmoc-L-propargylglycine (Fmoc-Pra-OH) and Fmoc-L-azidoalanine (Fmoc-Aza-OH) or Fmoc-L-azido-homoalanine (Fmoc-Aha-OH) provided the required functionality to prepare the triazole bridges. 1,2,3-Triazoles were then prepared as either the 1,4-isomer using a Copper-Catalyzed Azide-Alkyne Cycloaddition (CuAAC); or as the 1,5-isomer using a Ruthenium-Catalyzed Azide-Alkyne Cycloaddition (RuAAC) reaction. The progress of the reaction was monitored by IR spectroscopy (disappearance of the azide absorbance at $2110 \mathrm{~cm}-1$ ). The peptides were cleaved from solid support under acidic conditions and purified by reverse-phase HPLC. Of the four peptidomimetics produced, two retained good activity relative to U-II. U-II 1 give $\mathrm{pIC}_{50}=\mathrm{K}_{\mathrm{D}}$ of $9.36 \mathrm{nM}$ and peptidomimetics 2 and 3 also had essentially identical $\mathrm{IC}_{50}=\mathrm{K}_{\mathrm{D}}$ values of around $8 \mathrm{nM}$. Importantly, binding of compounds 2 and 3 but not U-II to UTR in CHOhUT cells was reversible.

With this successful application of the 1,5-triazole as a disulfide bond surrogate we decided to apply this strategy to a more challenging conotoxin peptide that incorporates two-disulfide bonds.

Conotoxin $\alpha$-GI 6 is a nAChR antagonist that elicits its effect at the skeletal neuromuscularjunction so has potential as a lead compound for the development of a new class of muscle relaxant.[6] Two analogues of globular native $\alpha$-GI were produced in which the two disulfide bonds were replaced in turn by the 1,5-triazole bridge designed previously.[7] The peptidomimetics were incubated with human muscle-type nAChRs and their activity determined in vitro by assessing antagonism of the $\mathrm{nAChR}$ mediated increase of $\left[\mathrm{Ca}^{2+}\right]$ in $\mathrm{CN} 21$ 
cells. One of the analogues 8, with the (Cys3-Cys13) disulfide bond replaced, retained full antagonist activity ( $\mathrm{IC}_{50} 8.2 \mathrm{nM}$ ) compared to native $\alpha$-GI 6 ( $\mathrm{IC}_{50} 9.8 \mathrm{nM}$ ). We next assessed the blood plasma half-life of the bioactive peptidomimetic vs the native $\alpha$-GI. Incorporation of the triazole bridge in place of the native disulfide significantly improves its plasma half-life by $\sim 10$ fold c.f. the native 6 . To investigate if the triazole acts as an accurate structural mimic of the disulfide bond we developed bespoke force field descriptions of the triazole mimetic that allowed us to determine the solution structure by NMR. Significant similarities in conformation between the mimetic and the peptidic bioactive toxin can be observed. It is evident that the conformation of the $\beta$-turn pharmacophore of the native peptide 6 is preserved in peptidomimetic 8. Our NMR structure supports a previously reported hypothesis that the pharmacophore of the $\alpha$-GI is located within the first half of the peptide as a $\beta$-turn.

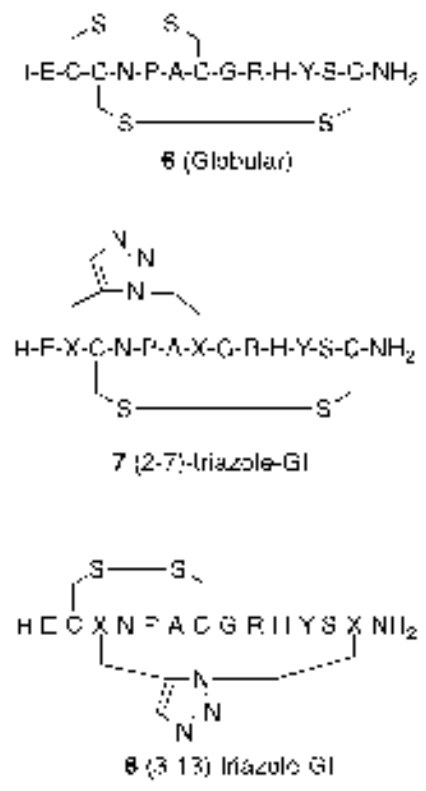

Figure 2: a-Conotoxin GI 6 and 1,5-triazole peptidomimetic analogues $7 \& 8$.

In summary, a highly accurate disulfide bond surrogate has been developed based on a 1,5-trizole that is synthetically tangible from commercially available staring materials. Given the effectiveness of the strategy described we believe this approach is broadly applicable to other peptides containing disulfide bonds that have potential for development as novel probes and therapeutics.

\section{References}

1. M. J. Adler, , A. G. Jamieson and Hamilton, A. D., Curr. Top. Microbiol. Immunol., 2011, 348, 1-23.

2. A. Gori, P. Gagni, S. Rinaldi, Chem. Eur. J. 2017, 23, 14987-14995.

3. M. Empting, O. Avrutina, R. Meusinger, S. Fabritz, M. Reinwarth, M. Biesalski, S. Voigt, G. Buntkowsky and H. Kolmar, Angew. Chem., Int. Ed., 2011, 50, 5207-5211.

4. N. D. Doan, T. T. M. Nguyen, M. Létourneau, K. Turcotte, A. Fournier and D. Chatenet, Br. J. Pharmacol., 2012, 166, 243-257.

5. S. Pacifico, A. Kerckhoffs, A. J. Fallow, R. E. Foreman, R. Guerrini, J. McDonald, D. G. Lambert, A. G. Jamieson, Org. Biomol. Chem., 2017, 15, 4704-4710.

6. I. G. Marshall , A. L. Harvey, Toxicon, 1990, 28, 231-234.

7. A. Knuhtsen, C. Whitmore, F. S. McWhinnie, L. McDougall, R. Whiting, B. O. Smith, C. M. Timperley, C. Green, K.I. Kinnear, A. G. Jamieson, Chem. Sci., 2018. 